\title{
Genetic diversity in European Pisum germplasm collections
}

\author{
R. Jing • M. A. Ambrose $\cdot$ M. R. Knox $\cdot$ P. Smykal \\ M. Hybl · Á. Ramos · C. Caminero · J. Burstin - G. Duc • \\ L. J. M. van Soest • W. K. Święcicki • M. G. Pereira • \\ M. Vishnyakova $\cdot$ G. F. Davenport $\cdot$ A. J. Flavell $\cdot$ T. H. N. Ellis
}

Received: 26 September 2011 / Accepted: 29 February 2012/Published online: 1 April 2012

(C) The Author(s) 2012. This article is published with open access at Springerlink.com

\begin{abstract}
The distinctness of, and overlap between, pea genotypes held in several Pisum germplasm collections has been used to determine their relatedness and to test previous ideas about the genetic diversity of Pisum. Our characterisation of genetic diversity among 4,538 Pisum accessions held in 7 European Genebanks has identified sources of novel genetic variation, and both reinforces and refines previous interpretations of the overall structure of genetic diversity in Pisum. Molecular marker analysis was based upon the presence/absence of polymorphism of retrotransposon insertions scored by a high-throughput
\end{abstract}

Communicated by I. Mackay.

Electronic supplementary material The online version of this article (doi:10.1007/s00122-012-1839-1) contains supplementary material, which is available to authorized users.

\section{R. Jing · A. J. Flavell}

Division of Plant Sciences, University of Dundee at JHI,

Invergowrie, Dundee DD2 5DA, UK

Present Address:

R. Jing

Institut für Biochemie und Biologie, Universität Potsdam, Karl-Liebknecht-Str. 24-25, Haus 26, 14476 Potsdam-Golm, Germany

M. A. Ambrose - M. R. Knox - T. H. N. Ellis

John Innes Centre, Norwich Research Park, Colney Lane,

Norwich NR4 7UH, UK

P. Smykal · M. Hybl

Agritec Plant Research Ltd., Zemedelska 2520/16,

78701 Sumperk, Czech Republic

P. Smykal

Department of Botany, Faculty of Sciences, Palacký University,

Slechtitelu 11, 78371 Olomouc, Czech Republic microarray and SSAP approaches. We conclude that the diversity of Pisum constitutes a broad continuum, with graded differentiation into sub-populations which display various degrees of distinctness. The most distinct genetic groups correspond to the named taxa while the cultivars and landraces of Pisum sativum can be divided into two broad types, one of which is strongly enriched for modern cultivars. The addition of germplasm sets from six European Genebanks, chosen to represent high diversity, to a single collection previously studied with these markers resulted in modest additions to the overall diversity observed, suggesting that the great majority of the total genetic diversity collected for the Pisum genus has now been described. Two interesting sources of novel genetic variation have been identified. Finally, we have proposed reference sets of core accessions with a range of sample

\footnotetext{
Á. Ramos

Centro para la calidad de los alimentos, INIA, Campus universitario, 42004 Soria, Spain

C. Caminero

Instituto Tecnológico Agrario, Consejería de Agricultura y Ganadería de la Junta de Castilla y León, Ctra Burgos, km 119, 47071 Valladolid, Spain

J. Burstin · G. Duc

Institut National de la Recherche Agronomique (INRA), UMR LEG, 17 rue de Sully-Building B1, Office 110, BP 86510, 21065 Dijon Cédex, France

L. J. M. van Soest

Centre for Genetic Resources, The Netherlands (CGN),

P. O. Box 16, 6700 AA Wageningen, The Netherlands

W. K. Święcicki

Institute of Plant Genetics, Polish Academy of Sciences, ul. Strzeszyńska 34, 60-479 Poznan, Poland
} 
sizes to represent Pisum diversity for the future study and exploitation by researchers and breeders.

\section{Introduction}

The diversity and taxonomy of Pisum has received considerable attention (Ellis et al. 1998; Pearce et al. 2000; Simioniuc et al. 2002; Vershinin et al. 2003; Baranger et al. 2004; Coyne et al. 2005; Tar'an et al. 2005; Espósito et al. 2007; Smýkal et al. 2008; Zong et al. 2009; Jing et al. 2010, Martin-Sanz et al. 2011; reviewed in Ellis 2011 and Smýkal et al. 2011). A consensus has emerged from these studies that the genus as a whole represents a broad continuum comprising two wild species Pisum elatius and Pisum fulvum (Vershinin et al. 2003) together with distinct domesticated groups or taxa, such as Pisum abysinicum and "cv. Afghanistan" (Westphal 1974; Young and Matthews 1982; Jing et al. 2010). The wild form, P. elatius, is remarkably diverse and almost as broad as the genus as a whole. $P$. elatius has also been taken to include other named groups that are not monophyletic or comprise taxa that are no more distinct than other $P$. elatius accessions (Vershinin et al. 2003; Jing et al. 2005; Ellis 2011).

A notable exception to this broad consensus was the description of an extensive collection of Pisum accessions focussed on diverse Chinese material (Zong et al. 2008, 2009). This has been discussed in relation to other germplasm (Smýkal et al. 2011) and while it appears that the Chinese material is indeed diverse (consistent with Jing et al. 2010), some features of the data such as the fragmentation proposed for P. fulvum does not seem to be well supported. A possible explanation for this fragmentation may be homoplasy, and this was discussed in relation to the relative mutation rates of different marker types by Ellis (2011).

\section{G. Pereira}

Instituto Nacional de Investigação Agrária, Apartado 6, 7350-951 Elvas, Portugal

M. Vishnyakova

N.I. Vavilov Institute of Plant Industry (VIR), Bolshaya

Morskaya Street 42-44, 190000 St. Petersburg,

Russian Federation

G. F. Davenport

Crop Informatics, 211 Malecon Armenariz, Miraflores,

Lima, Peru

\section{T. H. N. Ellis $(\bowtie)$}

Institute of Biological, Environmental and Rural Sciences, Aberystwyth University, Gogerddan,

Aberystwyth SY23 3EB, UK

e-mail: noe2@aber.ac.uk
The structure of genetic diversity in the John Innes Pisum germplasm collection has been described recently (Jing et al. 2010). This germplasm collection, which contains 1,200 Pisum sativum cultivars, 600 traditional landraces and 750 wild Pisum samples, together with genetic stocks and reference lines from other collections, is the most complete assembly of Pisum germplasm to be studied to date by marker analysis. A subset of the previously studied accessions that had been strongly assigned to STRUCTURE sub-groups by Jing et al. (2010) have been genotyped at 1,484 SSAP defined loci, while the new accessions were genotyped using 27 retrotransposon-based insertion polymorphisms (RBIPs) scored in high throughput by the tagged microarray marker (TAM) microarray method (Flavell et al. 2003). RBIPs are based on the insertions of LTR retrotransposons (mainly PDRl; Jing et al. 2005) and the use of PCR-based detection of the presence and/or the absence of single retrotransposon insertions by combining two primers flanking the insertion site with a single outward-priming transposon-specific primer (Flavell et al. 1998). Thus, RBIP yields codominant marker scores for the irreversible sequence differences, which are well suited to studying diversity at the genus level (Jing et al. 2010). The polymorphism data for the JI Pisum collection were analysed using both the program STRUCTURE (Pritchard et al. 2000) and multifactorial approaches (Perrier et al. 2003). The former approach yielded a stratified description of genetic diversity that comprised three primary STRUCTURE groups (groups 1-3) corresponding roughly to landrace, cultivar and wild samples, respectively. Sequential STRUCTURE analysis of these groups revealed sub-structuring into 14 subgroups, many of which correlated well with the taxonomic sub-divisions, domestication-related traits and/or geographical distributions for the corresponding samples.

Here, we extend the analysis of Pisum diversity by including a further 1,518 Pisum accessions selected from 6 other major European collections (Table 1). The main objectives of this study were to determine whether our earlier broad conclusions for the genetic structure of Pisum is supported by adding germplasm from across Europe, to determine the extent of distinctness of germplasm held in different germplasm centres and to propose a representative set of Pisum accessions for the future study and exploitation.

\section{Materials and methods}

Plant material

We analysed the 3,020 John Innes Pisum germplasm accessions described in (Jing et al. 2010), plus 9 duplicates 
Table 1 Participating European collections and details of the material analysed

\begin{tabular}{|c|c|c|}
\hline Genebank & $\begin{array}{l}\text { FAO } \\
\text { code }\end{array}$ & Number of accessions and focus of selection \\
\hline $\begin{array}{l}\text { ITACyL, Instituto Tecnológico Agrario de Castilla y León, } \\
\text { Valladolid, Spain }\end{array}$ & ESP109 & $\begin{array}{l}347 \text { comprising } 270 \text { Spanish landrace accession with sub- } \\
\text { accessions making up the balance }\end{array}$ \\
\hline $\begin{array}{l}\text { INRA, Station de Génétique et d'Amélioration des Plantes, Dijon, } \\
\text { France }\end{array}$ & FRA043 & $\begin{array}{l}360 \text { representing the French core collection with external } \\
\text { reference lines }\end{array}$ \\
\hline $\begin{array}{l}\text { CGN, Centre for Genetic Resources, the Netherlands, Wageningen, } \\
\text { NLD }\end{array}$ & NLD037 & 172 are landrace accessions originating from Asiatic highlands \\
\hline $\begin{array}{l}\text { Poznanska Hodowla Roslin, Plant Breeding Station, Wiatrowo, } \\
\text { Poland, POL } 004\end{array}$ & POL003 & $\begin{array}{l}364 \text { broad selection including wild accessions, landraces, } \\
\text { cultivars and genetic stocks }\end{array}$ \\
\hline $\begin{array}{l}\text { EAN-BANCO, Banco de Germoplasma, Genetica Estacao } \\
\text { Agronomica Nacional, Oeiras, Portugal }\end{array}$ & PRT005 & 52 Portuguese landraces accessions \\
\hline $\begin{array}{l}\text { N.I. Vavilov Research Institute of Plant Industry (VIR), St. } \\
\text { Petersburg, Russia }\end{array}$ & RUS001 & 305 cultivated forms including 116 from across Russia \\
\hline
\end{tabular}

and 1,518 accessions from 6 other European germplasm collections (listed in Table 1). Access to this extended germplasm was facilitated through the Working Group for Grain Legumes of the European Cooperative Programme for Crop Genetic Resources (ECPGR). The complete list of accessions is in Supplementary Table 1.

Plant growth and DNA preparation

Single plants for each accession were grown at each of the six germplasm centres, then dried leaf segments stored in silica gel were sent to a single location (Scotland) for DNA extraction by the Qiagen DNeasy 96 method.

RBIP markers and TAM microarray-based marker analysis

27 RBIP markers were selected from an original set of 45 (Jing et al. 2010) on the basis of their informativeness (allele frequencies in the JI collection), reliability and data quality (signal to noise ratio, $\mathrm{Cy} 3 / \mathrm{Cy} 5$ ratio). Marker scoring was as described in Jing et al. (2010), with the addition of a dye swap (hybridization of Cy3-labelled and Cy5-labelled probes to separately arrayed aliquots from the same PCR reaction set).

\section{SSAP markers}

PDR1 SSAPs are amplicons derived from TaqI digested genomic DNAs to which adaptors have been ligated. The PCR uses two base selective primers corresponding to the adaptor and a labelled retrotransposon primer directed towards the $3^{\prime} 156$ bp LTR that lacks TaqI restriction sites (Ellis et al. 1998). In the present study, these were screened as fluorescent markers on an ABI 3730 xl platform using 16 selective $P D R 1$ primer combinations carrying all possible 2-base $3^{\prime}$ extensions on the adaptor primer (Knox et al. 2009).
Genetic diversity data analysis

Genotypic scores were collated and analysed as described in Jing et al. (2010), with the Dice genetic distance measures used in this analysis calculated using DARwin5 (Perrier et al. 2003). The program STRUCTURE (Pritchard et al. 2000; Pritchard and Wen 2004) and the method of Evanno et al. (2005) were used to model potential relationships between accessions. Correlation analysis between STRUCTURE runs (Supplementary Figure 1) was performed as follows.

For each run $(r)$, each accession has a value of $Q_{\mathrm{rp}}$ corresponding to the presumed contribution of each proposed progenitor population $(p)$. The correlation between pairs of runs was calculated using Genstat v13 (Payne et al. 2008) as the average of the absolute value of all possible pairwise correlations of populations. Note that not all runs were equally well self-correlated as this depends on the correlation between populations within a run.

Multifactorial analysis (MFA) in DARwin5 (Perrier et al. 2003) involved calculating simple match scores for all pairs of markers, recorded as fractions of shared markers (Jing et al. 2010). The NJ tree (Fig. 1) was calculated from allele frequencies within the population sub-groups of Jing et al. (2010) in an Excel sheet. Correspondence analysis (Supplementary Figure 2) was performed using DARwin5 (Perrier et al. 2003) to calculate Dice genetic distances between pairs of accessions for the 45 or 27 genetic marker data sets.

\section{Geographical relationships}

Latitude and longitude data were plotted in Google Earth using . $\mathrm{kml}$ files (Supplementary Tables $2 \mathrm{a}$ and $2 \mathrm{~b}$ ) with the image simplified using Photoshop CS3. Great circle distances were calculated from $35^{\circ} 47^{\prime} \mathrm{N} 72^{\circ} 36^{\prime} \mathrm{E}$ using the relationship: $y_{\mathrm{i}}=2 \arcsin \sqrt{ }\left[\sin ^{2}(a / 2)+\cos \left(\theta_{\mathrm{c}}\right) \cos \left(\theta_{\mathrm{i}}\right) \sin ^{2}(b / 2)\right]$. 


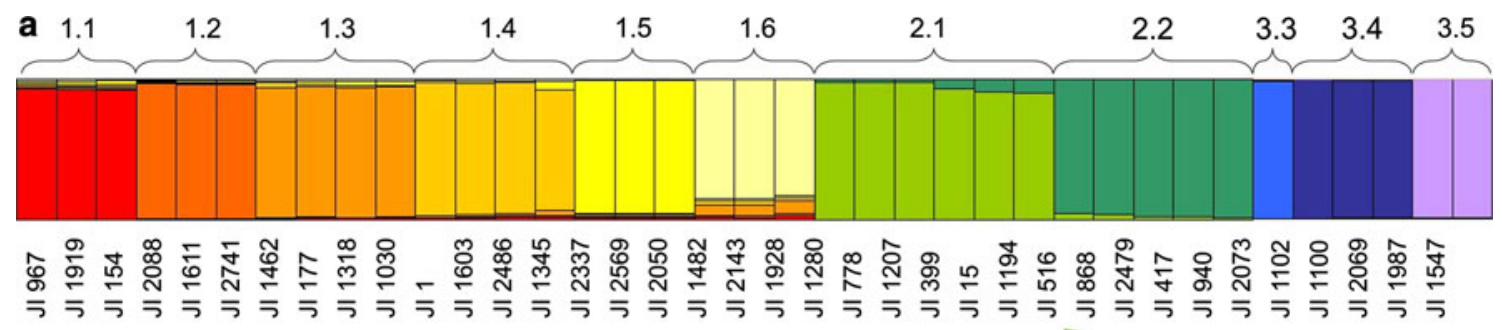

b

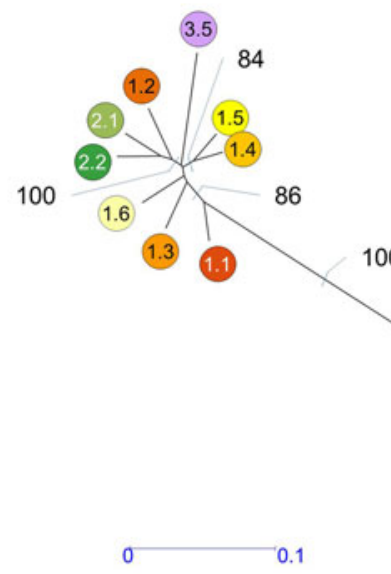

\section{c}

cv Afghanistan

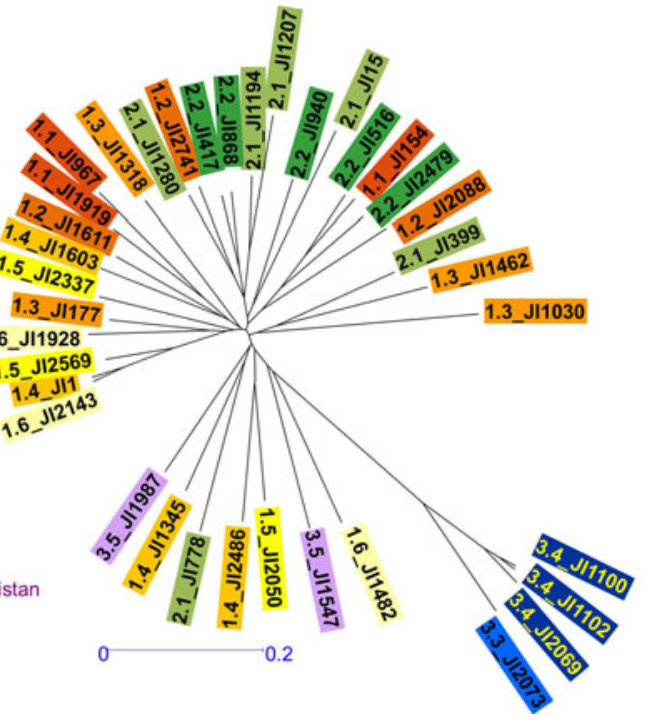

Fig. 1 SSAP analysis of strongly assigned accessions. a Assignment of $Q$ from Jing et al. (2010). b Neighbour joining (NJ) tree calculated using DARwin5 (Perrier et al. 2003). Allele frequency within the population sub-groups of Jing et al. (2010) was calculated in an Excel

The values $a$ and $b$ are given as: $a=\theta_{\mathrm{c}}-\theta_{\mathrm{i}}$ and $b=\varphi_{\mathrm{c}}-\varphi_{\mathrm{i}}$; all angles were in radians, where $\theta$ and $\varphi$ are latitude and longitude, $\theta_{\mathrm{c}}$ is the latitude of the reference point and $\theta_{\mathrm{i}}$ is the latitude of the sample.

Data storage and visualisation

The original RBIP data of 45 RBIP markers derived from Jing et al. 2010 are stored in the Germinate Pea database http://bioinf.scri.ac.uk/germinate_pea/app/. These include the 27 markers used in this study. Corresponding data for the 1,518 extra lines scored with these 27 RBIP markers have been stored in the same database.

\section{Selection of a representative set of accessions}

Accessions with high values of $Q$ corresponding to the STRUCTURE sub-groups of Jing et al. (2010) and the STRUCTURE groups and sub-groups from the analyses presented here were identified together with outlying accessions in the MFA; several accessions were identified in both procedures (Supplementary Table 1).

Core Hunter software (Thachuk et al. 2009) was used to select accessions for core collections using a multiobjective sheet and the majority consensus NJ tree with 100 bootstraps calculated. Bootstrap values higher than $80 \%$ are indicated. c The corresponding $\mathrm{NJ}$ tree for individual accessions is shown

measure, which consisted of an equally weighted contribution of Modified Rogers' distance, Cavalli-Sforza and Edwards distance and Shannon-Weaver index, Core subsets for seven accessions (the minimum core), and 5, 10, 20 and $30 \%$ of the full set of accessions were derived (Supplementary Table 1). To investigate the propensity of accessions to be sampled by Core Hunter, the $30 \%$ selection was resampled to yield an alternative $10 \%$ core. The overlap between the two $10 \%$ subsets is $280 / 453$, when 151 would be expected by chance alone (Table 2), indicating that Core Hunter shows a tendency to resample the same accessions.

\section{Results}

Partitioning Pisum accessions into groups

The analysis of Jing et al. (2010) partitioned 3,020 accessions of the John Innes (JI) Pisum collection into a hierarchical organisation comprising, three main groups which subdivided into 14 sub-groups, some of which were more clearly distinct than others. To test these assignments, a set of 37 P. sativum accessions that were strongly assigned to 
these STRUCTURE sub-groups (Fig. 1a) was selected for more detailed marker analysis. These were screened using 1,484 PDR1 SSAP markers (Ellis et al. 1998; Knox et al. 2009) of which 625 amplicons were found to be polymorphic in this data set. From this information, neighbour joining trees were constructed for the sub-groups and accessions (Fig. 1b, c) using the DARwin5 software (Perrier et al. 2003).

RBIP analysis of Pisum from European germplasm collections

A new sample set was assembled from major European Pisum germplasm collections, comprising 422 accessions from France, 368 accessions from Spain, 295 accessions from Russia, 212 accessions from Poland, 171 accessions from The Netherlands and 50 accessions from Portugal (Supplementary Table 1). DNAs from these samples were scored for 27 RBIP markers that were a subset of those 45 previously investigated (Jing et al. 2010). The resulting marker data were combined with the corresponding existing data for the same markers in the JI Pisum collection to produce a sample set of 4,547 containing 9 replicated samples. RBIP markers score the presence and absence of an insertion site simultaneously, however, that does not necessarily signify heterozygosity because either sequence may be duplicated. Here, and previously, such marker states are treated as missing data as discussed in Jing et al. (2010). In total, the allele calls were 42,339 occupied site alleles, 54,956 empty site alleles and 25,474 data points treated as missing data. This is a slightly lower frequency of missing data points than in the previous data set $\left(\chi^{2}=83\right)$. The frequency distribution of missing marker scores in the data set is shown in Supplementary Figure 3.

\section{Correspondence between data sets}

To test correspondence between the old and new data sets, two measures of pairwise genetic distance were made for 394 Pisum accessions previously scored, one $\left(\mathrm{D}_{45}\right)$ with the set of 45 markers and the other $\left(\mathrm{D}_{27}\right)$ with the set of 27 markers. These two values were strongly correlated ( $r=0.9$, Supplementary Figure 2).

\section{Bayesian analysis of population structure}

The program STRUCTURE (Pritchard et al. 2000; Pritchard and Wen 2004) has been widely used for the description of genetic variation, and analysis with this method formed the basis for the general conclusions drawn by Jing et al. (2010). Here, we undertook the same analysis with the 4,547 samples scored for 27 selected markers. As before (Jing et al. 2010), there was no strong indication of the most appropriate value of $K$, the number of proposed ancestral populations. The value $K=2$ partitioned the data set robustly, roughly separating group 2 of Jing et al. (2010) from the rest of the germplasm (data not shown), but failed to resolve the most distinct germplasm set, namely group 3 , which contains the large majority of wild and primitive cultivated Pisum germplasm of the JI Pisum germplasm collection.

At $K=3$, the correlation between the output of 20 independent STRUCTURE runs fell into 6 classes, according to a hierarchical cluster analysis of the inter-run correlations (Supplementary Figure 1a). Three of these classes, comprising half of the runs were more closely related to each other than the others and these were taken for further analysis (Supplementary Figure 1b, c).

The assignment of parentage to those accessions common to this study and Jing et al. (2010) was compared between the two data sets (Fig. 2). The overall correlations between the assignments of $Q$ for the corresponding groups are: $Q_{\mathrm{G} 1}, Q_{\mathrm{B}} 0.32, Q_{\mathrm{G} 2}, Q_{\mathrm{R}} 0.34$ and $Q_{\mathrm{G} 3}, Q_{\mathrm{G}} 0.95$, where $\mathrm{G} 1, \mathrm{G} 2$ and $\mathrm{G} 3$ are the main STRUCTURE groups of Jing et al. (2010) and $Q_{\mathrm{B}}, Q_{\mathrm{R}}$ and $Q_{\mathrm{G}}$ refer to the STRUCTURE groups of the extended data set (Supplementary Figure 1b). There were also weaker but significant correspondences between the two other groups $\left(Q_{\mathrm{B}}\right.$ and $\left.Q_{\mathrm{R}}\right)$ and sub-groups of these analyses (Fig. 2). Sub-group 1.1 of Jing et al. (2010) (the first sub-group along the $x$ axis, coloured red) is noticeably distinct and seems to correspond mainly to the components of $Q_{\mathrm{R}}$ in the new analysis and for high assignments to $Q_{\mathrm{R}}$ (Fig. 2).

The strong correspondence between group 3 and the small group $\left(Q_{\mathrm{G}}\right)$, identified in the present analysis, was examined further by repeating the STRUCTURE analysis on this subset of accessions, using the $K=6$ value derived for group 3 by Jing et al. (2010) [no other $K$ value was strongly suggested by STRUCTURE or the method by Evanno et al. (2005)]. 16 out of 20 STRUCTURE runs were very strongly correlated and the average of these is compared to the previous data in Fig. 3. There are three very clear correspondences, namely between the pairs G3.1 and 3B, G3.3 and 3C, and G3.6 and $3 \mathrm{E}$. The accessions previously assigned to subgroups G3.4 and G3.5 are distributed among sub-groups 3A, $3 \mathrm{D}$ and $3 \mathrm{~F}$ from the new study.

Multifactorial analysis of Pisum population structure

A genetic distance matrix was calculated for 4,532 of the 4,547 samples (15 accessions had high numbers of missing scores, such that some pairwise comparisons could not be calculated) and a multifactorial analysis (MFA) performed on it ("Materials and methods"). The results for the first two dimensions of the resulting MFA are shown in Fig. 4. There is a broad overlap in the distribution of the 


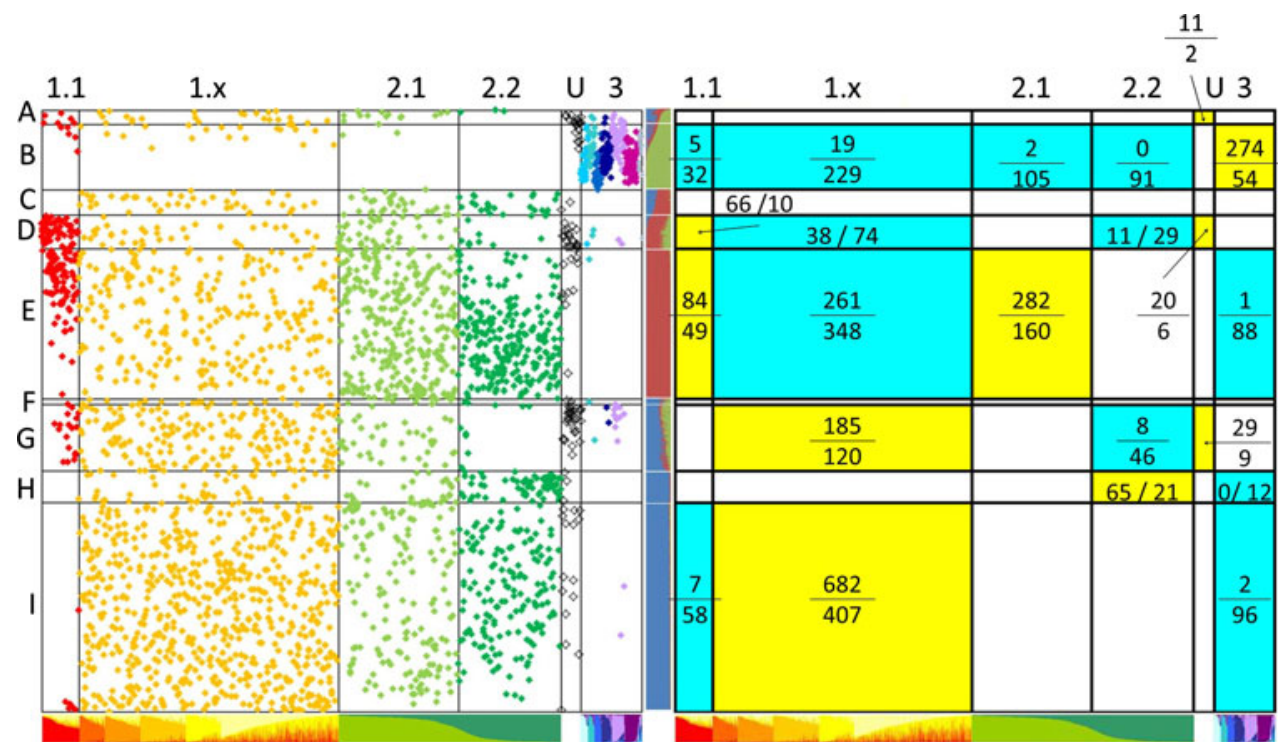

Fig. 2 Comparison of STRUCTURE analyses. The bottom horizontal ribbon (for both panels) shows the assignment to STRUCTURE sub-groups by Jing et al. (2010). The central vertical ribbon shows the assignment of $Q$ values with the data set from this study (see Supplementary Figure 1c). The upper green group is referred to as $Q_{\mathrm{G}}$ and the red-brown and blue groups as $Q_{\mathrm{R}}$ and $Q_{\mathrm{B}}$, respectively. Spots in the left panel graph indicate the locations of accessions in the two analyses. Observed versus expected numbers are indicated as a fraction for combinations of cells in the right panel, which are significantly different from expectation on the basis of a contingency test. Cells highlighted in yellow have significantly more accessions than expected and those highlighted in turquoise have fewer than expected
Fig. 3 Relationship between STRUCTURE groups containing exotic germplasm. Accessions assigned to the 'exotic' group $\left(Q_{\mathrm{G}}\right)$ of the $K=3$ analysis of this data set (the green group of Supplementary Figure 1b) were analysed by STRUCTURE and the most consistent assignment into six sub-groups is presented. The locations of accessions analysed by Jing et al. (2010) ( $x$ axis) are marked by points $(x, y)$ corresponding to their position in that and the analysis of the present data set ( $y$ axis). The diamond symbols at $(x, y)$ are colour coded according to the scheme of Jing et al. (2010) as indicated on the right. Taxonomic groups strongly represented in subgroups are indicated in brackets

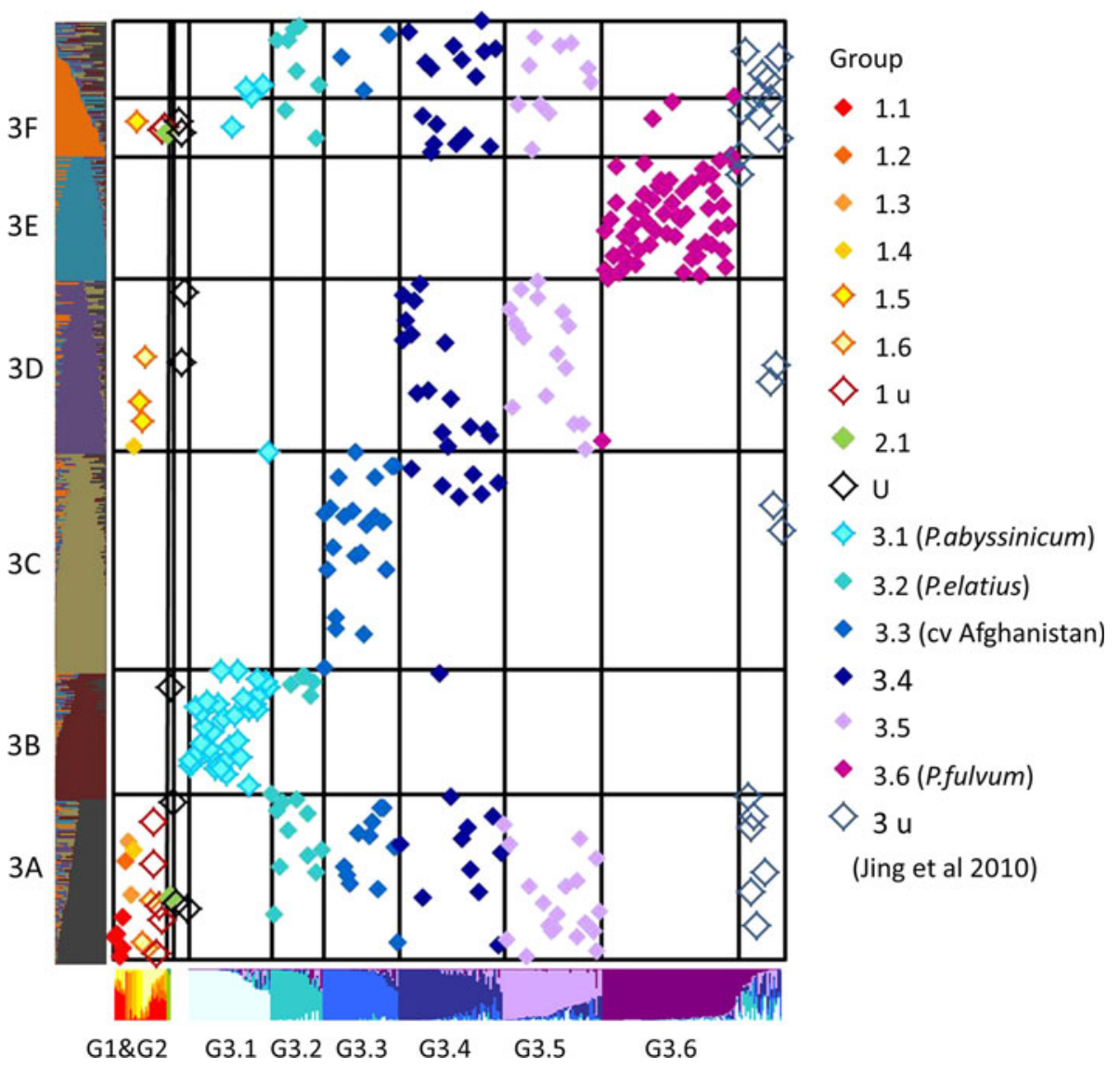


accessions in the new and old data set (Fig. 4a-c), but in the outer region of the plot (boxed in Fig. $4 \mathrm{~b}$ and highlighted in Fig. 4d, e) there is reciprocal excess or deficiency between the prior and new data. The region near coordinates $(0.4,-0.2)$ is enriched in new accessions (mainly from the Dutch germplasm collection) and some accessions assigned to Pisum humile. The region enriched in the old data set (Jing et al. 2010), near coordinates (0.2, 0.1 ), co-locates with many $P$. elatius and $P$. fulvum accessions (Fig. 4d, e).

A small number of accessions are peripherally located (Fig. 4f). Those in the upper portion of the multifactorial plot correspond to material from the Polish germplasm collection; four are annotated as $P$. abyssinicum (POZP17, POZP18, POZP20, POZP120) and one (POZP12) is assigned to $P$. elatius.

The outlying accessions at the lower part of the MFA (Fig. 4f) are from the old data set (Jing et al. 2010). Three of these (filled grey in the figure) have more that $50 \%$ missing marker data, so their location is probably unreliable. The others have less missing data and are presumably truly diverse at the combination of loci scored here.

Comparing samples from different germplasm collections

The distribution of accessions by germplasm collection is illustrated in Fig. 5. All of these collections contain much material from the central region of the plot, which includes modern cultivated $P$. sativum, and the peripheral germplasm we have analysed mainly derives from the UK (JIC), Dutch (NLD), French (FRA) and Polish (POL) collections. The Dutch accessions sampled (Fig. 5 NLD) are partitioned into two main genotypic groups and these are explored further in Fig. 6. Collection site information (latitude/longitude coordinates) is available for all of these accessions and these are plotted in Fig. $6 \mathrm{~b}$ (with the exception of a single Mexican accession identified as a blue spot in Fig. 6a). Almost all of the spots residing in the central region of the MFA plot in Fig. 6a correspond to

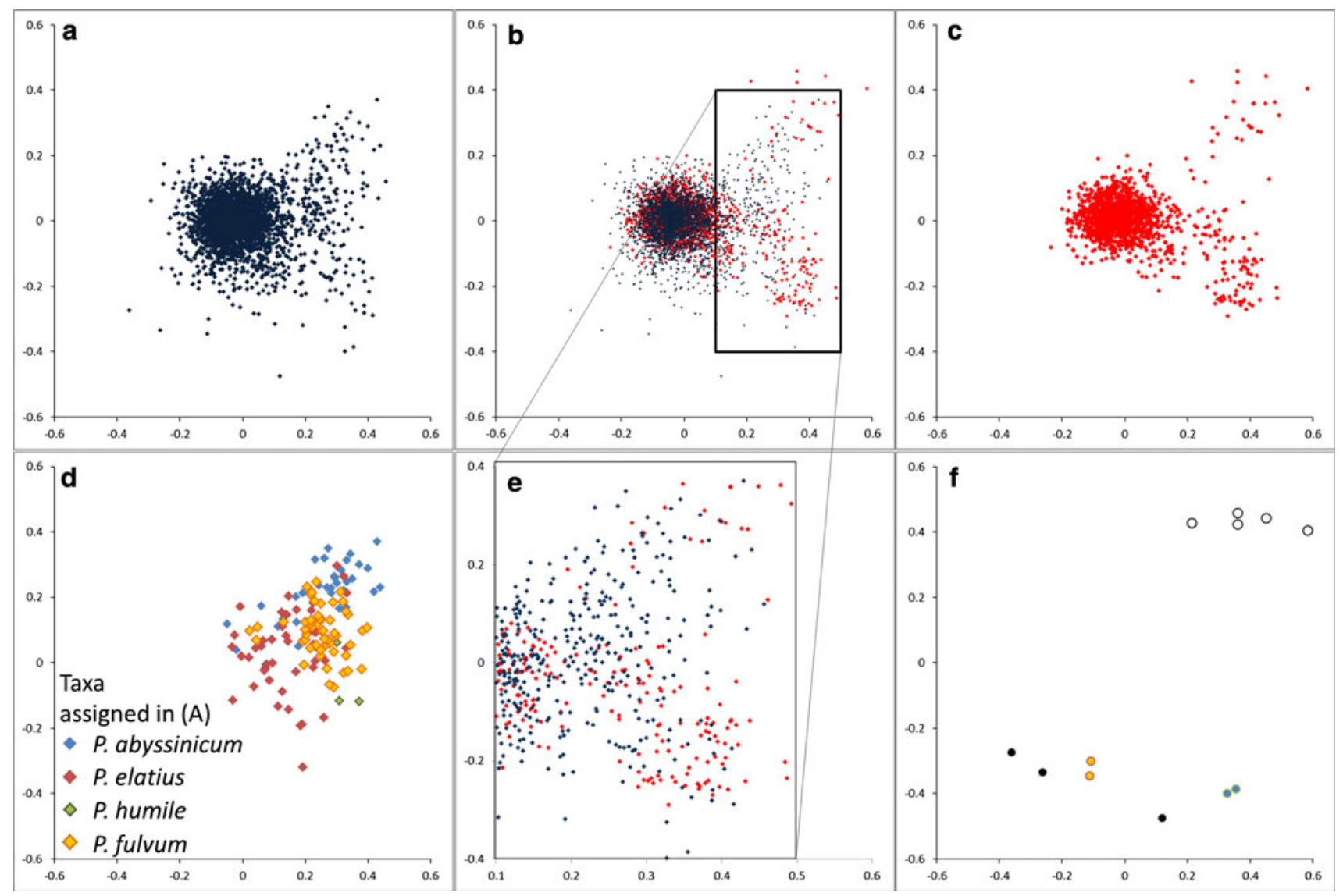

Fig. 4 Multifactorial analysis of Pisum diversity. Distribution of accessions in MFA: these two dimensions explain 7.24 and $3.61 \%$ of the variance, respectively (note that the variance is distributed over 4,532 dimensions). a Accessions previously analysed by Jing et al.
(2010) b All accessions in the present analysis. c Accessions new to this analysis. $\mathbf{d}$ Accessions in a assigned to four main taxa other than $P$. sativum. e Region centred near $(0.3,0)$. f Outlying points 

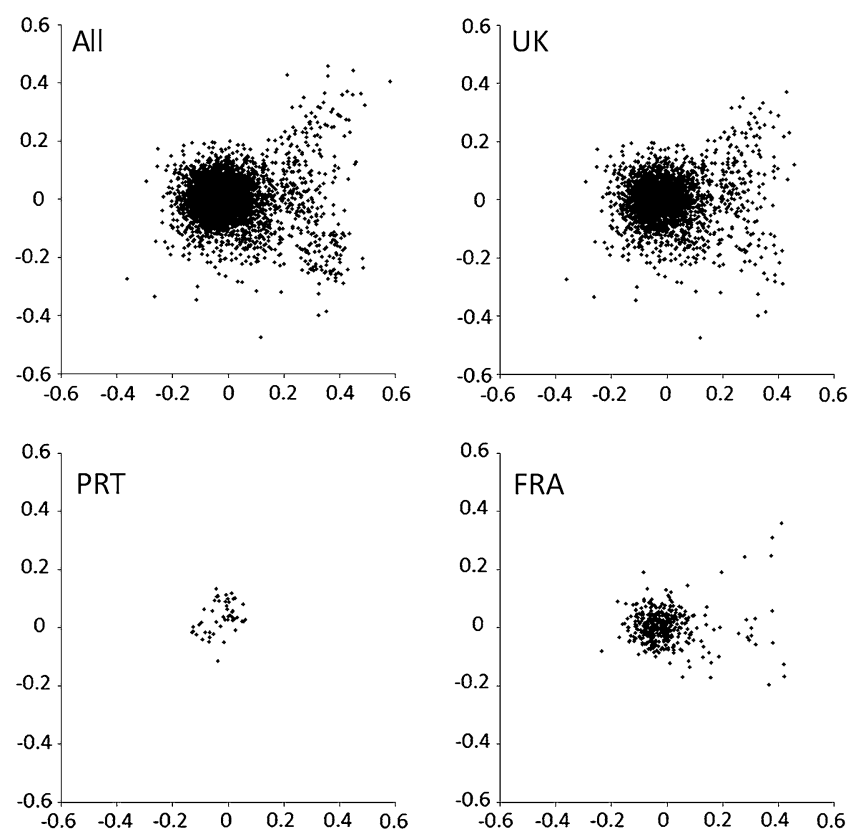

Fig. 5 Distribution of accessions by donor. This figure reproduces the MFA plot of Fig. 4, and shows the relative position of accessions obtained from various European germplasm collections. The codes are: All the complete data set, $U K$ the John Innes Pisum collection, $N L D$ the Dutch pea collection (Wageningen), POL the Polish pea

samples deriving mainly from Europe, Turkey and adjacent Middle East countries (yellow) and India (ringed, orange). In contrast, the green spots derive from germplasm that is mainly from the Himalayan foothills, mostly in northern Pakistan from the Konar River system. There are a few exceptions to these groupings; two of the Konar River accessions belong to 'Indian' genetic types and one Indian accession is of the 'Konar River' type (Fig. 6b, c).

If the accessions marked in green represented a selection from pea genotypes in the Indian sub-continent, then we would expect a measure of genetic distinctness correlated with the distance. The first component of the multifactorial plot clearly separates these accessions (Fig. 6a, $x$ axis), but when this is plotted against distance (Fig. 6c, $y$ axis) no clear relationship between genetic and physical distances is seen.

Identifying a representative subset of Pisum accessions for the future study

Two approaches were taken to identify subsets of accessions that represent the genetic diversity present in the germplasm studied here (Fig. 7). The first combined the STRUCTURE and multifactorial analyses in this and our previous studies (Jing et al. 2010). 14 sub-groups of accessions were identified in the previous study and here 3 groups have been identified; the most diverse of which has been further subdivided into 6 sub-groups, together giving
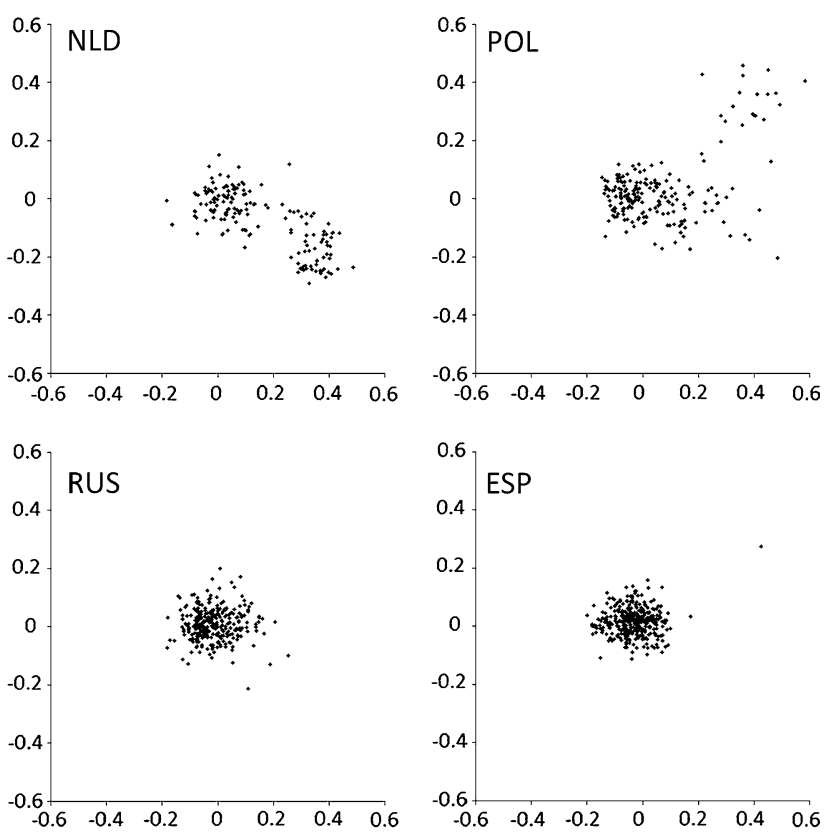

collection (Wiatrowo), PRT the Portuguese pea collection (Elvas), $F R A$ the French pea collection (Dijon), RUS the Russian pea collection (at the Vavilov Institute St. Petersburg), ESP the Spanish pea collection (Valladolid)

23 groups and sub-groups. Six accessions strongly assigned to each of these 23 groupings were selected by their high corresponding $Q$ values (Supplementary Table 1). This should correspond to 138 accessions, but the number was 134 because some individuals were selected from both a group and a sub-group. These were augmented with the 7 outliers in the MFA plot discussed above, to maximise the represented diversity, giving 141 accessions. The distributions of these accessions in the STRUCTURE and MFA plots are shown in Fig. 7b, $\mathrm{f}$.

The second approach for generating representative germplasm core subsets used the Core Hunter program (Thachuk et al. 2009; http://corehunter.org), which identifies subsets of representative accessions on the basis of maximising average genetic distance ("Materials and methods"). Core sub-sets for the minimum core of seven accessions 5, 10, 20 and $30 \%$ of the full set of accessions are shown in Fig. 7 (listed in Supplementary Table 1). To investigate the propensity of particular accessions to be sampled by Core Hunter, the $30 \%$ selection was resampled to yield an alternative $10 \%$ core in which 280 of the 453 were resampled when 151 would be expected by chance alone (Table 2).

The distribution of these sampled accessions with respect to each other within both STRUCTURE and MFA plots is shown in Fig. 7, and the analysis of the frequency with which individual accessions are represented in the different sets is presented in Table 2. The smaller sets (with 
Fig. 6 Geographic and genotypic partitioning of Dutch accessions. a Multifactorial plot (from Fig. 5 NLD), colour coded. b Location of assigned collection points for accessions. The pale blue spot in a corresponding to a Mexican accession is not shown. The yellow spots in the Indian subcontinent are marked as ringed symbols. For reference, high points in the Himalayan range are marked in black. The cluster of accessions from northern Pakistan is boxed in red and shown expanded to the right, with the mountainous region shaded grey. For scale, the distance between Jalalabad and Islamabad is $\sim 250 \mathrm{~km}$. c The centre of the group of accessions corresponding to the solid green spots tightly clustered in northern Pakistan is at $35^{\circ} 47^{\prime} \mathrm{N} 72^{\circ} 36^{\prime} \mathrm{E}$ near

Mahodand Lake south west of the Karakoram Mountains. The great circle distance from this point (in radians) for all accessions ( $y$ axis) is plotted against PC1 ( $x$ axis). Accessions are coloured yellow or green according to the two main groups in a. The yellow group is subdivided into ringed and solid colours according to the location shown in b. Three exceptions are: red an accession not clearly assigned to either yellow or green, blue a Mexican accession and the pale green ringed symbol that corresponds to an accession in the green group that was collected at a more southern location (indicated in b)

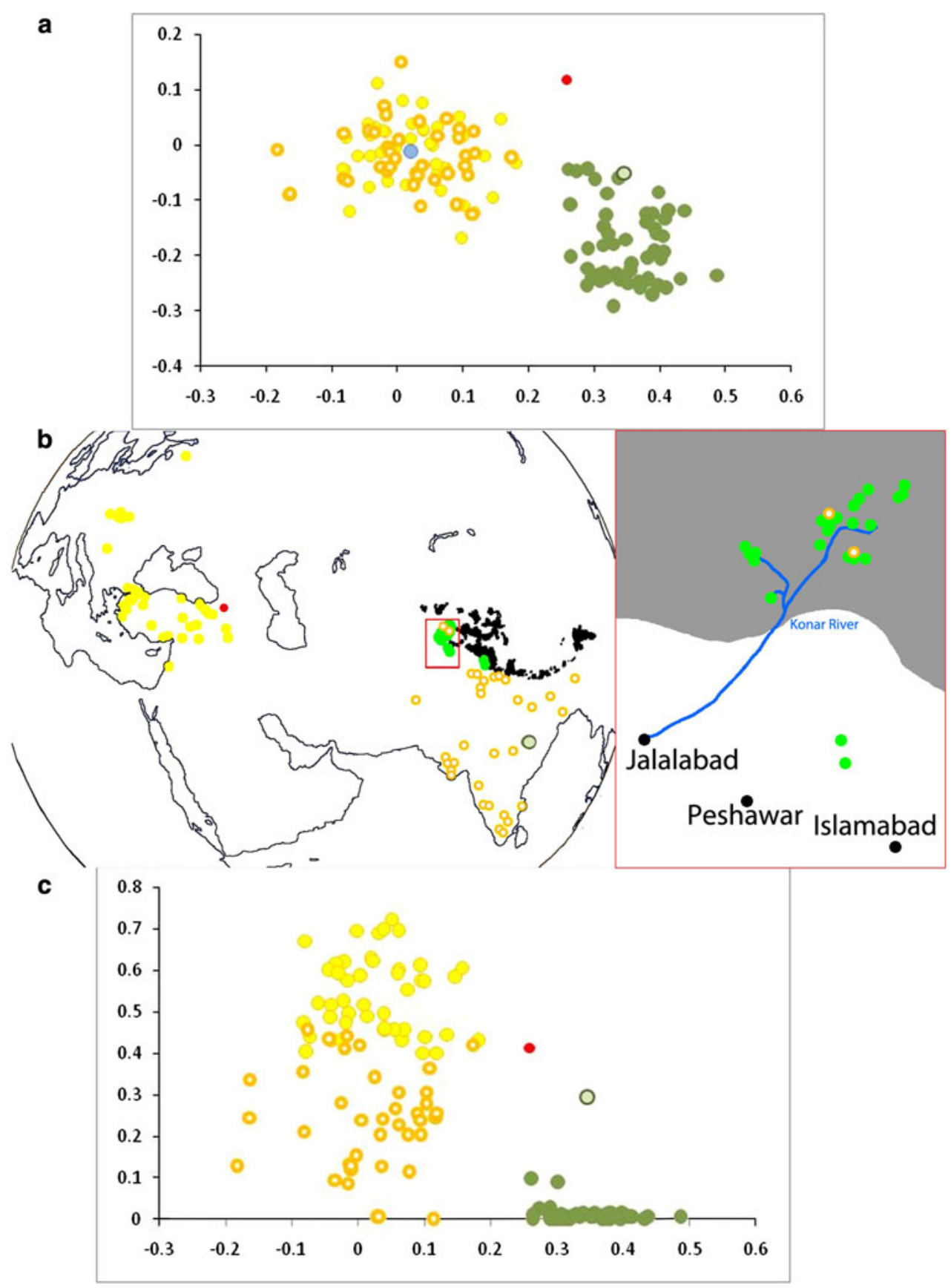

fewer than 500 members S, CH5 and CH10 in Table 2) selected accessions that were most likely to occur in other selections and the Core Hunter $5 \%$ set performed best by this measure (Table 2). These selections generally overrepresent alleles with respect to the data set as a whole; the most extreme is for 1006nr13 (AJ966283). The smaller suggested core samples tend to have the most extreme over-representation of rare alleles and the Core Hunter method has a greater over-representation than the STRUCTURE/MFA-based selection method.

\section{Discussion}

The main purpose of our study was to use molecular markers to determine the range of overlap, and extent of distinctness, of germplasm held in different collections. Our results show that the assignment of accessions to groups and sub-groups as presented by Jing et al. (2010) is broadly corroborated for a smaller set of accessions analysed with 12-fold more markers (Fig. 1). The SSAP markers used for this experiment share the property with 


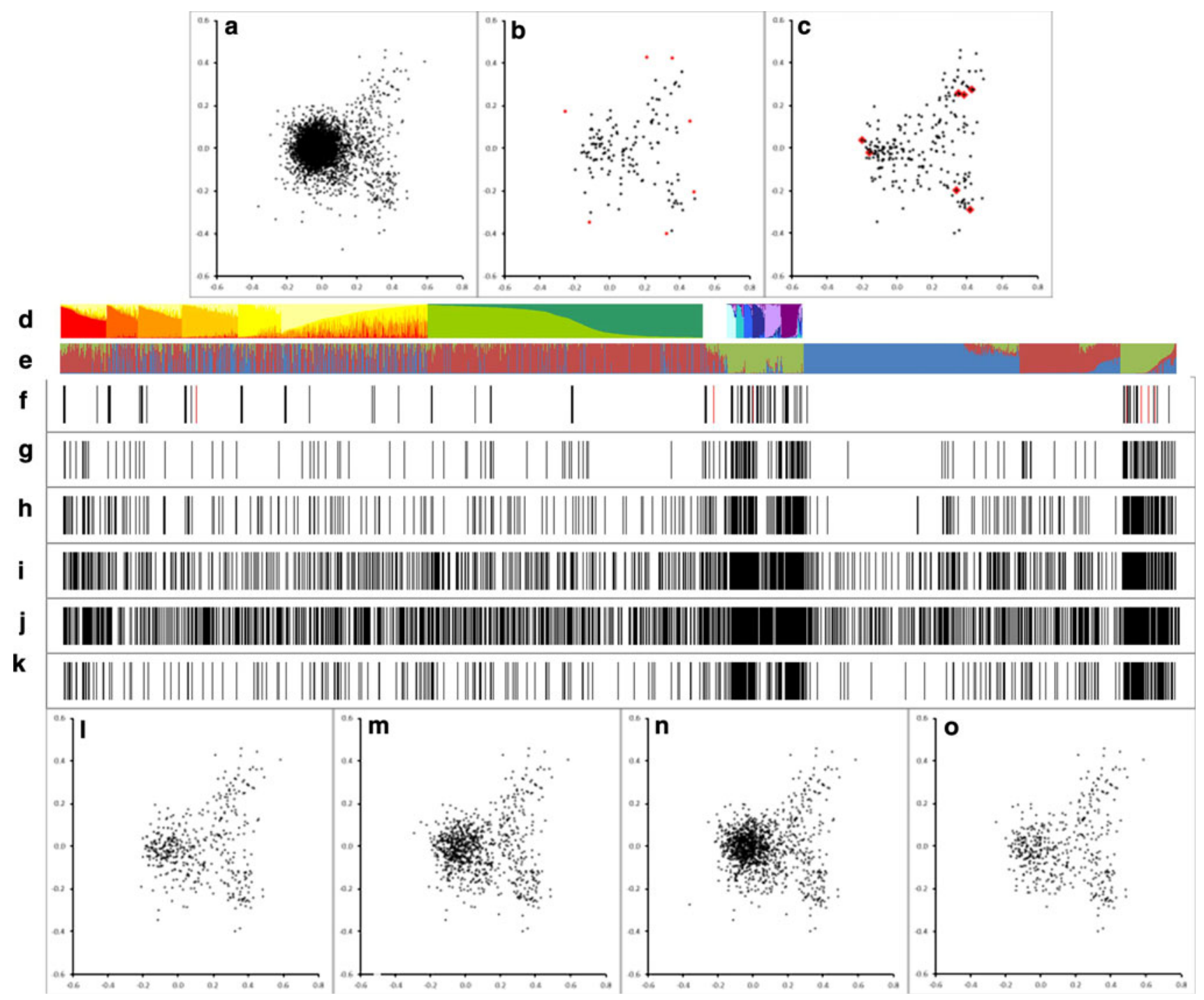

Fig. 7 Representative subsets of Pisum accessions. Multifactorial analysis (MFA) plots (a-c, l-o) and STRUCTURE assignments $(\mathbf{d}-\mathbf{k})$ of selected subsets of accessions are illustrated. The distribution of all accessions in the MFA space is shown in ' $a$ ' (identical to Fig. 4a). The assignments of accessions to STRUCTURE sub-groups of Jing et al. (2010) is shown in 'd', using their colour codes and accession order. e The assignment of accessions to three STRUCTURE groups identified here (blue $Q_{\mathrm{B}}$, red $Q_{\mathrm{R}}$ and green $Q_{\mathrm{G}}$ ). Those accessions common to $\mathbf{a}$ and $\mathbf{b}$ are in the order of Jing et al. (2010),

RBIPs of being based upon the insertion polymorphism of retrotransposons, but the latter is a codominant single locus approach and the former a dominant multi-locus approach (Waugh et al. 1997; Ellis et al. 1998) similar to transposon display (Van den Broeck et al. 1998). The SSAP analysis broadly supports the conclusions of Jing et al. (2010), notably for the accessions in sub-groups 3.3, 3.4 and 3.5; the close association of the sub-groups 2.1 and 2.2, and the lack of clear subdivision within group 1 except for subgroup 1.1. The similar results from these two marker but those on the right (new to this analysis) are in the order of Supplementary Figure 1c, with $Q$ assigned as the averages of panel A1. b, f 141 accessions sampled on the basis of STRUCTURE assignments (black) and MFA (red). c, g Samples selected by Core Hunter at $5 \%$ representation (black) with the seven accessions also in the minimum core highlighted in red. The 10, 20 and $30 \%$ Core Hunter selections are in $\mathbf{l}-\mathbf{n}$ (MFA) and $\mathbf{h}-\mathbf{j}$ (STRUCTURE), respectively. o, k $10 \%$ representation reselected from the $30 \%$ selection

approaches supports our assertion that the small number of markers used here has yielded reliable genetic diversity data for the 4,532 samples studied using them. Furthermore, the 27 RBIP marker set used reports essentially the same inter-accession distances as for Jing et al. (2010) suggesting that both measures report essentially the same features of genetic distances that distinguish these accessions, further reinforcing our approach. The slightly lower proportion of missing data in the data set we analysed suggests that the new data is at least as robustly scored as 
Table 2 Statistics of inclusion rates of accessions in different representative samples

\begin{tabular}{|c|c|c|c|c|c|c|}
\hline Sample & $\mathrm{S}$ & $\mathrm{CH} 5$ & $\mathrm{CH} 10$ & $\mathrm{CH} 20$ & $\mathrm{CH} 30$ & Resample \\
\hline \multirow[t]{2}{*}{ Number } & 141 & 226 & 453 & 906 & 1,359 & 453 \\
\hline & & $\mathrm{CH} 5$ & $\mathrm{CH} 10$ & $\mathrm{CH} 20$ & $\mathrm{CH} 30$ & Resample \\
\hline \multirow[t]{4}{*}{ S vs.: } & Observed & 43 & 63 & 81 & 84 & 59 \\
\hline & Expected & 7.01 & 14.05 & 28.09 & 42.14 & 47.00 \\
\hline & $\chi^{2}$ & 184.84 & 170.59 & 99.63 & 41.58 & 3.06 \\
\hline & & & $\mathrm{CH} 10$ & $\mathrm{CH} 20$ & $\mathrm{CH} 30$ & Resample \\
\hline \multirow[t]{4}{*}{ CH5 vs.: } & Observed & & 193 & 197 & 187 & 177 \\
\hline & Expected & & 22.52 & 45.03 & 67.55 & 75.33 \\
\hline & $\chi^{2}$ & & $1,290.89$ & 512.86 & 211.25 & 137.21 \\
\hline & & & & $\mathrm{CH} 20$ & $\mathrm{CH} 30$ & Resample \\
\hline \multirow[t]{4}{*}{ CH10 vs.: } & Observed & & & 336 & 339 & 280 \\
\hline & Expected & & & 90.26 & 135.39 & 151.00 \\
\hline & $\chi^{2}$ & & & 669.03 & 306.19 & 110.21 \\
\hline & & & & & $\mathrm{CH} 30$ & Resample \\
\hline \multirow[t]{3}{*}{ CH20 vs.: } & Observed & & & & 534 & 331 \\
\hline & Expected & & & & 270.78 & 302.00 \\
\hline & $\chi^{2}$ & & & & 255.86 & 2.78 \\
\hline
\end{tabular}

The samples compared are coded as follows: S the 141 accessions selected on the basis of STRUCTURE groups and MFA outliers, CH5, CH10, $\mathrm{CH} 20$ and $\mathrm{CH} 30$ are the sets selected by Core Hunter as representing 5, 10, 20 and $30 \%$ of the accessions, respectively. The column headed 'Resample' corresponds to 453 accessions sampled by Core Hunter from the 1,359 in CH30. The row observed is the number of accessions occurring in both samples as on the left and in the column header. The row expected is the number expected on the basis of the relative sample sizes and total number of accessions. The row $\chi^{2}$ is the Chi-squared value of the contingency test of the number observed versus expected

The average of the observed/expected ratio for the three combinations for each sample is: $\mathrm{S}, 3.87$; $\mathrm{CH} 5,5.46 ; \mathrm{CH} 10,4.82 ; \mathrm{CH} 20,3.24 ; \mathrm{CH} 30$, 2.31; and for resampled, 1.64, suggesting that, normalised for the number of accessions sampled, the accessions sampled by Core Hunter at $5 \%$ representation are those most likely to be resampled by another method

the previously analysed data set, even though the number of loci scored is reduced. This is most likely because of technology improvement, in particular the use of a dye swap during the TAM hybridization step for all markers (Jing et al. 2007a).

We have used two main approaches to analyse the marker data, namely STRUCTURE and MFA. The three STRUCTURE groups of accessions studied here correspond approximately to the three groups of Jing et al. (2010) with a strong correspondence between group 3 (coloured blue/purple in Fig. 2a) and the smallest of the three groups identified here, coloured green $\left(Q_{\mathrm{G}}\right)$, reflecting the robust assignment of accessions to the 'wild' material of STRUCTURE group 3 and the less reliable assignment for the cultivated germplasm which has lower intrinsic genetic diversity. Although the assignment of accessions to sub-groups within STRUCTURE group 1 is not robust (Jing et al. 2010), and was considered poorly resolved, the distinctness of the sub-group 1.1 is again notable (Figs. 2, 3, 7).

Although we tried to maximise the genetic distinctiveness of the new germplasm sampled here, much of the germplasm collected is closely related to cultivated $P$. sativum (Fig. 4). This occupies the central region of the multifactorial plots (Figs. 4, 5) and its dense packing in this region gives the impression that these accessions are highly similar, but this is to some extent misleading. Cultivated $P$. sativum displays lower genetic diversity than wild and primitive cultivated Pisum, but the large majority of marker alleles described in the latter can be found in the former (Vershinin et al. 2003). The major difference between these two germplasm classes is the variety of marker combinations (haplotypes) in the wild germplasm.

This study is consistent with the previous analyses and again emphasises the distinctness of $P$. sativum cv. Afghanistan and the taxa fulvum and abyssinicum. P. elatius accessions are concentrated in $3 \mathrm{~A}$ and $3 \mathrm{~F}$, along with accessions previously assigned to G1.1, G3.3, G3.4 and G3.5, consistent with $P$. sativum having been domesticated from this taxon (Vershinin et al. 2003, Jing et al. 2010). These are, therefore, good candidates for extant representatives of $P$. sativum that are most closely related to the wild taxa (Jing et al. 2010).

Two new classes of accessions, deriving from the Dutch (Fig. 6) and Polish collections (Supplementary Figure 4), respectively, have also been identified. In some ways, this is a relatively modest addition to the total genetic diversity available and underscores the breadth of the JI collection, which has captured the majority of the genetic diversity studied here. Nevertheless, this result underlines the need 
for caution in interpreting the genetic diversity of species on the basis of data from a single collection, even if it is large. It is clear that the JI collection contains the majority of the collected diversity available, but it nevertheless is poorly represented in two sectors of the global diversity of Pisum and it is possible that other sectors of Pisum diversity remain to be described.

In the Polish collection, the most unusual material corresponds mainly to populations assigned to $P$. abyssinicum or $P$. elatius. These new accessions seem remarkably diverse relative to previous studies which concluded that the genetic diversity of $P$. abyssinicum is strikingly compact (Ellis et al. 1998; Pearce et al. 2000; Vershinin et al. 2003, Baranger et al. 2004; Jing et al. 2005, 2007b, 2010; Smýkal et al. 2011) indicating that these are distinct from ' $P$. abyssinicum' as previously used (Supplementary Figure 4). The pattern of relatedness among these accessions suggests three possibilities: (1) these accessions may represent novel genetic variation within $P$. abyssinicum, (2) these accessions may be admixed $P$. abyssinicum, or (3) these accessions may be misclassified. Whichever of these is the case, the accessions are worthy of further analysis and have been included in our representative sets of accessions (Fig. 7; Supplementary Table 1; Supplementary Figure 4).

The novel Dutch accessions analysed here occupy a region of the MFA plot that contains JI material mainly from Turkey, Afghanistan, Iran and North India. This geographical location was recognised by Vavilov (1992) as a potential secondary centre of diversity for Pisum. These accessions are novel genotypes and, therefore, of particular potential interest to breeders and geneticists looking for new sources of variation in this species group. This result also suggests that those accessions held at VIR, sampled by Vavilov and colleagues, but not included in this study would be worthy of detailed molecular marker analysis in the future. The distinctness of these accessions from the Konar River system seems clear, but detailed examination of relatedness and geographical distance is consistent with Indian accessions from nearby showing some degree of admixture with the former that declines with distance from the collecting area (not shown). We conclude that these accessions represent distinct germplasm and note that samples of these are included in all of the approaches we have taken to identify a representative set of accessions (Fig. 7).

There is a lower abundance of accessions of wild species, and the diverse wild and exotic germplasm of group 3 of Jing et al. (2010), in the newly analysed accessions (Fig. 7d) and the corresponding green-coloured STRUCTURE group in Fig. 7e is highly sampled by all the methods used to identify a representative subset of Pisum accessions for the future study.

We conclude from these studies that the overall genetic diversity within the genus Pisum has been confirmed as a broad continuum with some substructure. Of special note are those accessions of $P$. sativum that are markedly distinct from cultivated types. cv. Afghanistan was recognised as an 'ecotype' by Young and Matthews (1982) and it is the source of the sym 2 allele that confers specificity on the symbiotic relationship with rhizobia, plus other distinct loci and traits consistent with it having adapted to a distinct environmental niche. However, its distinctness from other $P$. sativum has not been widely appreciated and it is clear from our study that it is almost as distinct as P. fulvum. This cv. Afghanistan class corresponds mainly to a single sub-group (G3.3) in our previous analysis and splits into two classes $3 \mathrm{~A}$ and $3 \mathrm{C}$ in the new data (Fig. 3). While this split does not correlate well to polymorphism at the Sym2 locus (Young and Matthews 1982), it does correspond to polymorphism at several of the loci studied here (1794-2, $281 \times 16,399 \times 131$ and 45x31). Although cv. Afghanistan is genetically coherent, it is also genetically diverse and the sym 2 allele is distributed between at least two sub-types of cv. Afghanistan.

The inclusion of novel genetic material from northern Pakistan (Fig. 6) has changed the genetic composition of the group of accessions that includes G3 of Jing et al. (2010). It is, therefore, not surprising that our STRUCTURE analysis of this $Q_{\mathrm{G}}$ partitions the $P$. sativum accessions in a slightly different way from G3.3. Nevertheless, the other two sub-groups of $P$. sativum accessions (G3.4 and G3.5) identified to be most distinct from cultivated types by Jing et al. (2010) have again been identified here, although they show a tendency to occur together (Fig. 3, sub-group 3D).

These data have been used to identify accessions with the potential of representing most of the genetic diversity in European Pisum germplasm collections in a far smaller number of accessions (Fig. 7; Supplementary Table 1). No two uses of such selections will necessarily have the same imperatives or requirements, so we have confined ourselves to using broad genetic diversity (assessed by two different approaches) to suggest several core collections of different sizes from seven individual accessions to more than a thousand. A recent search for novel $a$ alleles (Hellens et al. 2010) based on the STRUCTURE groups of Jing et al. (2010) identified a rare allele, and this accession was found in all of our core selections, apart from the 5 and $10 \%$ Core Hunter selections, suggesting that our sets of accessions can provide useful genetic diversity.

These selections generally over-represent rare alleles, and a tendency to equalise allele frequencies would be expected for methods sampling distinct haplotypes equally. The Core Hunter samples had the strongest normalisation of allele frequencies and this is the most extreme in the smaller samples. Core Hunter shows a tendency to resample the same accessions. Thus, we are confident that 
we have characterised the large majority of extant genetic diversity, which is held Pisum in germplasm collections and identified genetic variation not previously characterised, with corresponding geographical information on the locations of sources of this additional genetic novelty.

We have used a modest number (27) of markers for the analysis of a relatively large number of accessions $(4,538)$ and compared that to the analysis of smaller subsets $(3,020$ and 37) with larger numbers of markers (45 and 1,484) noting that the relationships between accessions were broadly similar. The 45 marker set was noticeably easier to analyse than the 27 marker data set suggesting that something of the order of 50 markers would be sufficient for the analysis of ca. 5000 accessions. Analysis of the data set with the complementary model building (STRUCTURE) and analytical (MFA) approaches was helpful in attracting attention to specific subsets of the data. The marker type we employed (retrotransposon insertion sites) is useful because the insertions are not reversible and occur at a relatively low rate (ca. $5 \times 10^{-7}$ per generation, Jing et al. 2005). This marker method is not widely used but neutral SNPs (although reversible) having a mutation rate of the order of $10^{-9}$ per generation should, in contrast to more rapidly evolving sites, have a low homoplasy rate and be suitable for extensive germplasm surveys.

Acknowledgments This work was supported by the European Commission Framework program VI Grain Legumes Integrated Project (FP6-2002-FOOD-1-506223). P.S. and M.H. were supported by MSM 2678424601. Financial support for the maintenance of the JI Pisum collection from Defra is also gratefully acknowledged. We thank J. Hofer for discussions and comments on the manuscript.

Open Access This article is distributed under the terms of the Creative Commons Attribution License which permits any use, distribution, and reproduction in any medium, provided the original author(s) and the source are credited.

\section{References}

Baranger AG, Aubert G, Arnau G, Lainé AL, Deniot G, Potier J, Weinachter C, Lejeune-Hénaut J, Lallemand J, Burstin J (2004) Genetic diversity within Pisum sativum using protein- and PCRbased markers. Theor Appl Genet 108:1309-1321

Coyne CJ, Brown A, Timmerman-Vaughan GM, McPhee KE, Grusak MA (2005) Refined USDA-ARS pea core collection based on 26 quantitative traits. Pisum Genet 37:3-6

Ellis THN (2011) Pisum. In: Kole C (ed) Wild crop relatives: genomic and breeding resources (legume crops and forages). Springer, New York, pp 237-248

Ellis THN, Poyser SJ, Knox MR, Vershinin AV, Ambrose MJ (1998) Polymorphism of insertion sites of Ty1-copia class retrotransposons and its use for linkage and diversity analysis in pea. Mol Gen Genet 260:9-19

Espósito MA, Martin EA, Cravero VP, Cointry E (2007) Characterization of pea accessions by SRAP's markers. Sci Hortic 113:329-335
Evanno G, Regnaut S, Goudet J (2005) Detecting the number of clusters of individuals using the software STRUCTURE: a simulation study. Mol Ecol 14:2611-2620

Flavell AJ, Knox MR, Pearce SR, Ellis THN (1998) Retrotransposonbased insertion polymorphisms (RBIP) for high throughput marker analysis. Plant J 16:643-650

Flavell AJ, Bolshakov VN, Booth A, Jing R, Russell J, Ellis THN, Isaac P (2003) A microarray-based high throughput molecular marker genotyping method: the tagged microarray marker (TAM) approach. Nucleic Acids Res 31:e115

Hellens R, Moreau C, Lin-Wang K, Schwinn KE, Thomson SJ, Fiers MWEJ, Frew TJ, Murray SR, Hofer JMI, Jacobs JME, Davies $\mathrm{KM}$, Allan AC, Bendahmane A, Coyne CJ, TimmermanVaughan GM, Ellis THN (2010) Identification of Mendel's white flower character. PLoS One 10:e13230

Jing R, Knox MR, Lee JM, Vershinin AV, Ambrose MJ, Ellis THN, Flavell AJ (2005) Insertional polymorphism and antiquity of PDR1 retrotransposon insertions in Pisum species. Genetics 171:741-752

Jing R, Bolshakov VI, Flavell AJ (2007a) The Tagged Microarray Marker (TAM) method for high throughput detection of single nucleotide and indel polymorphisms. Nat Protoc 2:168-177

Jing R, Johnson R, Seres A, Kiss G, Ambrose MJ, Knox MR, Ellis THN, Flavell AJ (2007b) Gene-based sequence diversity analysis of field pea (Pisum). Genetics 177:2263-2275

Jing R, Vershinin A, Grzebyta J, Shaw P, Smykal P, Marshall D, Ambrose M, Ellis THN, Flavell A (2010) The genetic diversity and evolution of field pea (Pisum) studied by high throughput retrotransposon based insertion polymorphism (RBIP) marker analysis. BMC Evol Biol 10:44

Knox M, Moreau C, Lipscombe J, Baker D, Ellis N (2009) Highthroughput retrotransposon-based fluorescent markers: improved information content and allele discrimination. Plant Methods $5: 10$

Martin-Sanz A, Caminero C, Jing R, Flavell AJ, Perez de la Vega M (2011) Genetic diversity among Spanish pea (Pisum sativum L.) landraces, pea cultivars and the world Pisum sp. core collection assessed by retrotransposon-based insertion polymorphisms (RBIPs). Span J Agric Res 9:166-178

Payne RW, Murray DA, Harding SA, Baird DB, Soutar DM (2008) GenStat for windows (11th edition) introduction. VSN International, Hemel Hempstead

Pearce SR, Knox M, Ellis THN, Flavell AJ, Kumar A (2000) Pea Ty1-copia group retrotransposons: transpositional activity and use as markers to study genetic diversity in Pisum. Mol Gen Genet 263:898-907

Perrier X, Flori A, Bonnot F (2003) Data analysis methods. In: Hamon P, Seguin M, Perrier X, Glaszmann JC (eds) Genetic diversity of cultivated tropical plants. Enfield, Science Publishers, Montpellier, pp 43-76

Pritchard JK, Wen W (2004) Documentation for STRUCTURE software Version 2

Pritchard JK, Stephens M, Donnelly P (2000) Inference of population structure using multilocus genotype data. Genetics 155:945-959

Simioniuc D, Uptmoor R, Friedt W, Ordon F (2002) Genetic diversity and relationships among pea cultivars revealed by RAPDs and AFLPs. Plant Breed 121:429-435

Smýkal P, Hýbl M, Corander J, Jarkovský J, Flavell A, Griga M (2008) Genetic diversity and population structure of pea (Pisum sativum L.) varieties derived from combined retrotransposon, microsatellite and morphological marker analysis. Theor Appl Genet 117:413-424

Smýkal P, Kenicer G, Flavell AJ, Kosterin O, Redden RJ, Ford R, Zong X, Coyne CJ, Maxted N, Ambrose MJ, Ellis THN (2011) Phylogeny, phylogeography and genetic diversity of the Pisum genus. Plant Genet Resour 9:4-18 
Tar'an B, Zhang C, Warkentin T, Tullu A, Vandenberg A (2005) Genetic diversity among varieties and wild species accessions of pea (Pisum sativum L.) based on molecular markers, and morphological and physiological characters. Genome 48:257-272

Thachuk C, Crossa J, Franco J, Dreisigacker S, Warburton M, Davenport GF (2009) Core Hunter: an algorithm for sampling genetic resources based on multiple genetic measures. BMC Bioinformatics 10:243

Van den Broeck D, Maes T, Sauer M, Zethof J, Peter De Keukeleire P, D’Hauw M, Van Montagu M, Gerats T (1998) Transposon Display identifies individual transposable elements in high copy number lines. Plant J 13:121-129

Vavilov NI (1992) Origin and geography of cultivated plants (translated by Löve D). Cambridge University Press, Cambridge. ISBN 0-521-40427-4

Vershinin AV, Allnutt TR, Knox MR, Ambrose MJ, Ellis THN (2003) Transposable elements reveal the impact of introgression, rather than transposition, in Pisum diversity, evolution and domestication. Mol Biol Evol 20:2067-2075
Waugh R, McLean K, Flavell AJ, Pearce SR, Kumar A, Thomas BT, Powell W (1997) Genetic distribution of BARE-1 retrotransposable elements in the barley genome revealed by sequencespecific amplification polymorphisms (S-SAP). Mol Gen Genet 253:687-694

Westphal E (1974) Pulses in Ethiopia, their taxonomy and agricultural significance. Verslag landbouwkundig onderzoek, Wageningen

Young JPW, Matthews P (1982) A distinct class of peas (Pisum sativum L.) from Afghanistan that show strain specificity for symbiotic Rhizobium. Heredity 48:203-210

Zong X, Guan JP, Wang SM, Liu QC (2008) Genetic diversity among Chinese pea (Pisum sativum L.) landraces as revealed by SSR markers. Acta Agron Sin 34:1330-1338

Zong X, Redden RJ, Liu Q, Wang S, Guan J, Liu J, Xu Y, Liu X, Gu J, Yan L, Ades P, Ford R (2009) Analysis of a diverse global Pisum sp. collection and comparison to a Chinese local collection with microsatellite markers. Theor Appl Genet 118: 193-204 